\title{
Semi-analytical study on the generic degeneracy for galaxy clustering measurements-ERRATUM
}

\section{Alejandro Guarnizo, Luca Amendola, Martin Kunz and Adrian Vollmer}

doi: http://dx.doi.org/10.1017/S1743921314010771, Published by Cambridge University Press, 1 July 2015.

Keywords. cosmology: observations, cosmology: theory, methods: statistical, surveys

The proof corrections supplied by the author were not made to the final version of the article (Guarnizo, Amendola, Kunz and Vollmer, 2015).

We apologise to the authors and to the readers for this omission.

After (2.6) on page 348 , the statement "being $\bar{S}=\bar{A} \bar{R}$." should read

"being $\bar{S}=\bar{A} \bar{R}$. and $P_{1}=\bar{R} / \bar{A} . "$

Improved versions of the figures are also presented below.

\section{References}

Guarnizo, A., Amendola, L., Kunz M., \& Vollmer, A. 2015, in A. Heavens, J-L. Starck \& A. Krone-Martins (eds), Statistical Challenges in 21st Century Cosmology, Proc. IAU Symposium No. 306, 347-350 

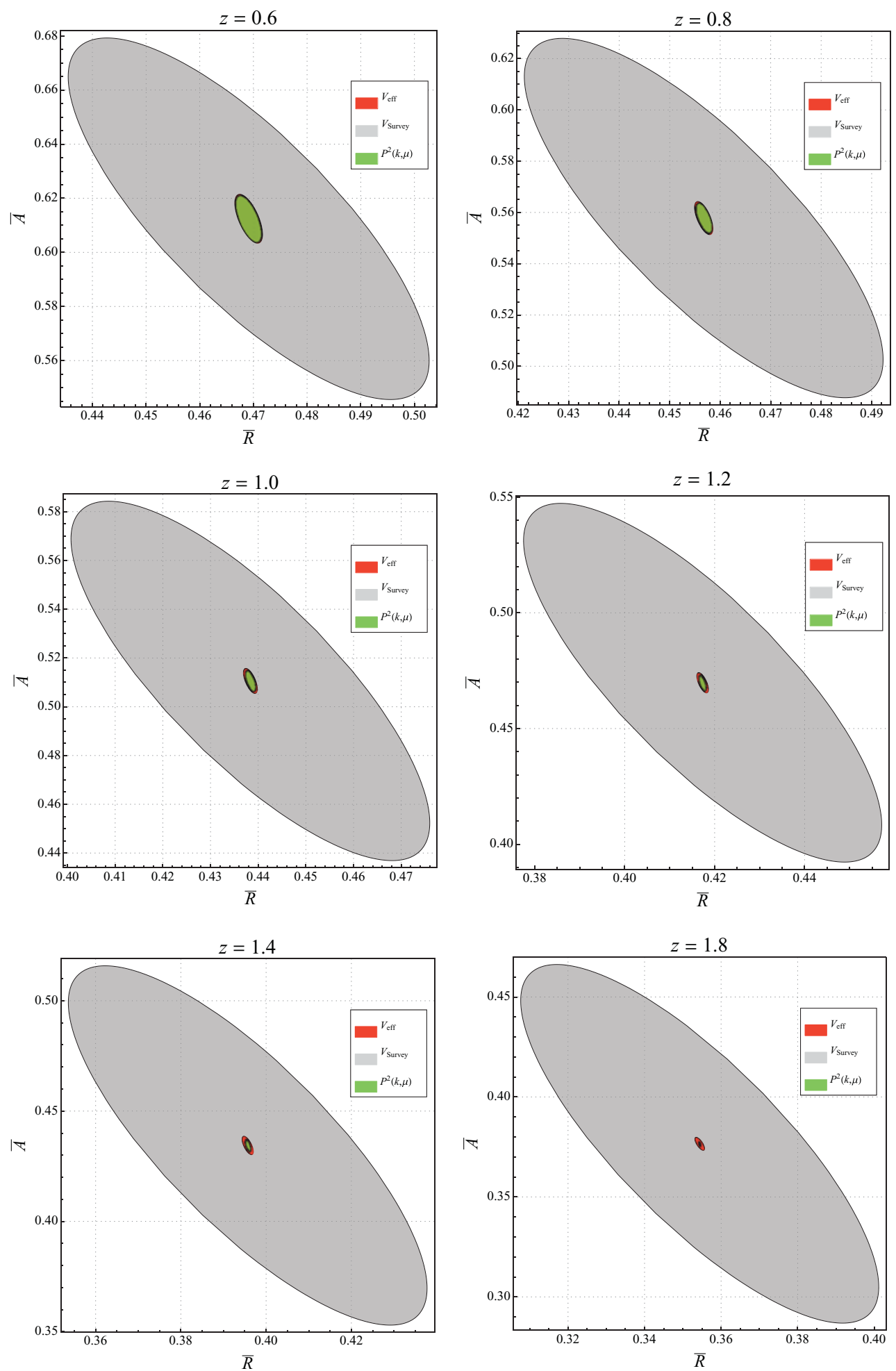

Figure 1. Confidence contours for $\bar{A}$ and $\bar{R}$ in the three cases: $V_{\text {eff }}, V_{\text {eff }} \approx V_{\text {survey }}$, and $V_{\mathrm{eff}} \approx P(k, \mu)^{2}$. 